\title{
Mapping the Aetiology and Antimicrobial Resistance Patterns of Maternal Bacterial Infection in Sub-Saharan Africa: A Systematic Review and Meta-Analysis Protocol
}

Chikondi Isabel Joana Chapuma ( $\sim$ cchapuma@mlw.mw)

Malawi-Liverpool-Wellcome Trust Clinical Research Programme https://orcid.org/0000-0002-33633205

Charlotte Van der veer

Malawi-Liverpool-Wellcome Trust Clinical Research Programme

Edward J M Monk

Malawi-Liverpool-Wellcome Trust Clinical Research Programme

Apatsa Selemani

University of Malawi Kamuzu College of Nursing: University of Malawi College of Medicine

David Kulapani

University of Malawi Kamuzu College of Nursing: University of Malawi College of Medicine

Catherine Dunlop

University of Birmingham Barnes Medical Library: University of Birmingham College of Medical and

Dental Sciences

Nicholas Feasey

Malawi-Liverpool-Wellcome Trust Clinical Research Programme

David Lissauer

Malawi-Liverpool-Wellcome Trust Clinical Research Programme

Protocol

Keywords: Maternal, Bacterial infection, Microbiology, Sub-Saharan Africa, AMR

Posted Date: December 29th, 2021

DOI: https://doi.org/10.21203/rs.3.rs-1194785/v1

License: (c) (1) This work is licensed under a Creative Commons Attribution 4.0 International License.

Read Full License 


\title{
MAPPING THE AETIOLOGY AND ANTIMICROBIAL RESISTANCE PATTERNS OF MATERNAL BACTERIAL INFECTION IN SUB-SAHARAN AFRICA: A SYSTEMATIC REVIEW AND META-ANALYSIS PROTOCOL
}

\author{
AUTHORS

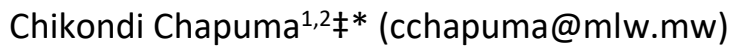 \\ Charlotte Van der veer ${ }^{1,2} \neq$, (cvanderveer@mlw.mw) \\ Edward J M Monk¹, (emonk@mlw.mw) \\ Apatsa Selemani ${ }^{3}$, (aselemani@kuhes.ac.mw) \\ David Kulapani ${ }^{3}$, (dkulapani@kuhes.ac.mw) \\ Catherine Dunlop ${ }^{4}$, (catherinedunlop@nhs.net) \\ Nicholas Feasey ${ }^{1,3,5}$, (nicholas.feasey@lstmed.ac.uk) \\ David Lissauer ${ }^{1,2}$ (dlissauer@mlw.mw)
}

\section{INSTITUTION NAMES AND ADDRESES}

1. Malawi Liverpool Wellcome Trust. P.O. Box 30096, Chichiri, Blantyre 3, Malawi, C. Africa

2. Women's and Child's Health, Institute of Life Course and Medical Sciences, University of Liverpool, William Henry Duncan Building, 6 West Derby Street, Liverpool L7 8TX, United Kingdom.

3. Kamuzu University of Health Science, Private Bag 360, Chichiri, Blantyre 3, Malawi.

4. Institute of Metabolism and Systems Research, University of Birmingham, IBR Tower, Level 2, College of Medical and Dental Sciences, Edgbaston , Birmingham B15 2TT, United Kingdom.

5. Liverpool School of Tropical Medicine, Pembroke Place Liverpool, L3 5QA UK, United Kingdom.

‡ Shared first authorship,

* Corresponding author 


\section{ABSTRACT}

Background: Over two-thirds of global maternal deaths occur in Sub-Saharan Africa (SSA), with more than 200,000 deaths per year. Maternal sepsis causes $10 \%$ of these deaths, twice the proportion observed in high-income countries. In SSA, limited access to diagnostic microbiology facilities poses difficulties in promptly identifying and managing maternal infection and sepsis. This protocol describes a systematic review and meta-analysis that aims to summarize available data on the main bacterial agents causing maternal infections and their antibiotic susceptibility in SSA.

Methods: Three electronic databases will be searched: MEDLINE, Embase and African Journals Online. Our search strategy will combine terms relating to laboratory-confirmed bacterial infection, pregnancy, postnatal period and SSA. We will include observational studies describing maternal bacterial infection's aetiology and antimicrobial resistance patterns in SSA. Two authors will perform study selection, data extraction and quality assessment. A third author will be consulted to resolve disagreements if they arise.

We will summarize the proportion (and 95\% confidence intervals) of samples testing positive for the most common bacteria and, depending on the data's availability and heterogeneity, examine results by country and/or region. If possible, we will describe trends over time and differentiate aetiological organisms and resistance/sensitivities by maternal infection sources. We will also undertake subgroup analyses based on HIV status, the invasive and non-invasive status of the infection, SSA sub-regions and mortality if there is adequate information to make such subgroup analysis feasible.

Discussion: Data on the microbiologic outcomes for maternal infections in SSA are likely fragmented and not fully representative due to the limited availability of microbiology diagnostics and geographical differences in clinical and laboratory practices. If this is the case, policies and programme strategies to guide treatment and identify antimicrobial resistance threats in SSA settings will be challenging to target. Our systematic review aims to provide a comprehensive summary of the available data, describe the main organisms causing maternal infection and their sensitivities, and identify areas that require further research.

Prospero ID: CRD42021238515 
KEYWORDS

Maternal

Bacterial infection

Microbiology

Sub-Saharan Africa

AMR

Version 1.0 


\section{BACKGROUND}

Over the past 25 years, there has been a 44\% decrease in Maternal Mortality Ratio (MMR) globally (1). This decrease in MMR is short of the Millennium Development Goals 2015 5a that targeted an MMR reduction of $75 \%$ (2). Therefore, there is an increasing need to strengthen efforts to reduce maternal mortality if the new Sustainable Development Goals target, to reduce MMR to less than 70 per 100,000 live births by 2030 is to be met (Target 3.1 ) (3).

The burden of maternal deaths is the highest in Sub-Saharan Africa (SSA) compared to the rest of the World (4)(5). Over two-thirds of global maternal deaths occur in SSA, with at least 200,000 deaths per year (6)(1). Of these deaths, up to $10 \%$ are due to infection which is twice the proportion observed in high income countries (7). The problem's origins lie in the low quality of care areas, including inconsistent infection prevention, poor infection treatment, delayed sepsis diagnosis, and inadequate sepsis management. In addition, limited availability of validated diagnostics (culture and sensitivity) poses difficulties in prompt identification and management of maternal infection in SSA (8). Therefore, prioritizing research in this arena to describe the existing landscape of maternal infections in this region is essential to guide simple strategies to prevent morbidity and mortality from maternal infections. It also has the potential of reducing maternal death rates globally.

Data on the microbiologic outcomes for non-invasive and invasive bacterial maternal infections can inform policies (programme strategies and treatment guidelines) and identify antimicrobial resistance (AMR) threats in SSA. However, there are challenges in estimating maternal sepsis burden due to differences in its definitions (9)(10)(11). Notably, there is a broad variation in the description of sepsis and infections in obstetrics. Furthermore, there is heterogeneity in the range of infections considered. Some definitions include only infections from the genital tract, whilst others have a broader meaning and include infections from other organ systems, such as pneumonia or incidental infections (malaria). The World Health Organization (WHO) proposes a new definition for maternal sepsis which we will use for this systematic review to describe any "symptomatic maternal bacterial infection". It spans across the pregnancy period spectrum, including the antenatal, peripartum, postpartum, and post-abortion periods (up to 42 days of pregnancy termination). It also incorporates an extensive host of infectious morbidities, including genital tract infections (e.g., chorioamnionitis and endometritis), extra-genital infections (e.g., mastitis, breast abscess, pyelonephritis, tetanus), as well as other maternal infections complicating pregnancy, childbirth, and the puerperium (e.g., HIV, malaria, sexually transmitted 
infections (STIs), urinary tract infections (UTIS) and other infectious diseases (10). The second challenge is that, despite significantly higher maternal infection-related mortality, the body of knowledge on maternal infections in this SSA is scarce because to date, there is no systematic review that summarizes the underlying microbiological agents causing maternal infection in SSA, nor their resistance patterns.

Therefore, using the WHO definition of maternal infection and sepsis, this systematic review will not only summarise the available data on the main bacterial agents causing maternal infection and their susceptibility to antibiotics. It will also assess availability and representativeness of the data. 


\section{METHODS}

\section{Protocol registration}

This is a systematic review protocol for observational studies describing microbial agents causing maternal infection and their susceptibility to antibiotics in SSA, if available. We prepared this protocol following the Preferred Reporting Items for Systematic Review and Meta-analysis Protocols (Appendix 1). This protocol is registered with the International Prospective Register of Systematic Reviews on March 2021 (registration number: CRD42021238515).

\section{Criteria for considering studies for this review}

Types of studies

We will include any observational study describing the aetiology and antimicrobial resistance patterns of maternal bacterial infection in SSA. These will consist of cohort studies, case-control studies, and crosssectional studies.

\section{Inclusion criteria}

This review will consider any studies that evaluate symptomatic laboratory-confirmed bacterial infection that is in keeping with the WHO definition of maternal infection/sepsis among pregnant and postpartum women (up to 42 days after birth). This review will include only studies evaluating data from SSA.

\section{Exclusion criteria}

We will exclude papers that contained erroneous, incomplete or internally inconsistent data; or assessed the diagnostic accuracy of any test using only positive samples and not in the clinical context of suspected maternal infection. We will also exclude studies that only describe maternal colonization of bacteria rather than infection. For example, we anticipate many studies that evaluate maternal colonization of Group B streptococci (GBS) in the context of neonatal infection. We will also exclude screening studies that do not include women who fulfil the WHO definition, for example studies that evaluate the prevalence of sexually transmitted infections among women attending routine clinical check-ups. Finally, we will exclude studies reporting on only one type of bacteria as this may skew proportional estimates.

\section{Search methods for identification of studies}

Version 1.0 


\section{Electronic search strategy}

To identify studies from SSA, we will conduct a comprehensive literature search through three databases from relevant disciplines: MEDLINE, Embase and African Journals Online. Using Boolean operators, our search strategy will combine terms relating to laboratory-confirmed bacterial infection, pregnancy and SSA.

Preliminary search strategies for MEDLINE are presented in Appendix 2. The final search strategy will be developed with the support of information experts and approved by the project team. If necessary, we will revise the search strategy to include any subject headings or high-level terms ineligible articles. The search will not contain letters or editorials. We will use the "humans" and "female" and "age" filters. We will attempt to translate non-English articles. We will also search the systematic review registries for ongoing reviews. Finally, we will contact experts to determine if any essential studies were omitted.

\section{Selection of studies}

Two independent authors (CC and CVD) will screen titles and abstracts with aid from a librarian (AS). If at least one of the authors approves the study, we will obtain the full-text report. In both stages, we will compare the results against eligibility criteria. We will resolve disagreements through discussion, and if we cannot reach an agreement, a third author (DL) will decide to select the study. We will provide reasons for excluding articles on a PRISMA flow diagram illustrating the screening process.

\section{Data analysis extraction and synthesis}

\section{Data collection}

Two authors will independently pilot a data extraction template (CC and CVD) developed in Microsoft Excel and, if necessary, update the template after several iterations. This template will capture: 1) identification details of the study: title, language, authors, year of publication, the country, region, setting (urban or rural), study design, study inclusion and exclusion criteria; 2) Participant characteristics: participants' age (median and range), gestation period, co-morbidities (for example HIV status) and sample size of the study; 3) Exposure of interest: type of bacterial infection (invasive or noninvasive), source of infection, when the invasive bacterial infection occurred (antenatal/postnatal); and 4) Outcomes of interest: number and type of samples taken, number of samples positive; the diagnostic method used, antimicrobial susceptibility testing results and method used, maternal outcomes (if reported). If disagreements in template design arise, a third author will be consulted (DL). 
For extraction, one of the authors will enter the data from each study into Microsoft excel (CC/EM), and the data will be checked by a second author (CVD). For studies published only as abstracts, we will attempt to contact the study correspondence authors for further information. For study reports containing little details on methods and results, we will contact the authors to obtain further information on these elements. Disagreements regarding the data extracted will be resolved by discussion and, if necessary, by consulting a third review author (DL).

\section{Quality assessment and risk of bias assessment}

To assess reporting completeness and quality of included studies, two authors will independently assess included studies according to Strengthening the Reporting of Observational Studies in Epidemiology and the Newcastle- Ottawa Scale, respectively (Appendix 3 and 4). Two authors (CC/CVD/EM) will apply the completeness and quality assessment checklist for observational studies to each included study independently.

\section{Data analysis}

\section{Descriptive measurements}

We will describe the most common bacterial organisms causing invasive and non- invasive maternal infection and their susceptibility to antimicrobials if available. Depending on the data's availability and heterogeneity, we will break down results per country or region and describe trends over time and differentiate aetiological organisms and resistance/sensitivities by maternal infection sources. In the context of clinical suspicion of maternal infection, we will summarize the proportion (and 95\% confidence intervals) of samples testing positive for the most common bacteria.

\section{Subgroup and sensitivity analyses}

Conditional to the data that will be found, we will undertake subgroup analyses based on HIV status, the invasive and non-invasive status of the infection, sub-regions of SSA and mortality (secondary objective). If insufficient numbers are available for the subgroups the analyses will be limited to descriptive analyses only. We will calculate the $\mathrm{I}^{2}$ statistic to measure heterogeneity. Due to the broad inclusion criteria, we expect that there will be substantial heterogeneity $\left(I^{2}>50 \%\right)$.

\section{Meta-analysis}


In such a review with broad definition and exclusion criteria, the $\mathrm{I}^{2}$ may be broad due to clinical heterogeneity. However, presenting this evidence as a forest plot is still valid. Therefore, we plan to undertake a meta-analysis using a random-effects model on data pooled from proportion estimate results from studies, if the data is appropriate, and present the results in a forest plot.

\section{DISCUSSION}

To the best of our knowledge, there is no systematic review on this subject to date. A Systematic review on invasive bacterial infections in neonates from SSA by Okomo et al. in 2019 that used similar methodology to our proposed review (12) identified Staphylococcus aureus, Klebsiella species and Group B streptococcus as the dominant pathogens causing respectively pneumonia, sepsis and meningitis among SSA neonates. Our systematic review on maternal infection will provide insight on whether obstetric infections in SSA are caused by pathogens similar to those causing neonatal infections. Our systematic review on maternal infections will also look at invasive and non-invasive pathogens causing maternal infections according to the WHO definition of maternal infection (10).

Due to the broadness of the definition and different clinical practices in different regions in SSA, we expect to see heterogeneity between the papers. However, if appropriate we will still intend to perform a meta-analysis of our data to assess for clinically relevant findings. Therefore, to mitigate anticipated clinical heterogeneity across the studies, we have focused the research question on the prevalence of invasive and non-invasive bacterial maternal infections and not include data on viral, fungal or other types of infectious agent (13). In addition, we will explicitly describe the research question, the literature research, the study selection, the data abstraction, quality assessment of the studies included, and data analysis in the review manuscript so that our findings are reproducible. We anticipate several confounders (HIV status, the invasive and non-invasive status of the infection, sub-regions of SSA); therefore, we will if possible, perform sub-analyses, but limit these to only on the sub-groups anticipated and named in this protocol. We also expect many articles reporting GBS ( Streptococcus agalactiae) in the target population(14)(15). GBS is a bacterial infection found in the female genital tract that rarely causes harm or symptoms to women. It, however, can cause neonatal infections if transmitted from mother to neonate during birth. Therefore, we will only include GBS papers if they report on maternal infection outcomes. Finally, we will also exclude papers reporting urinary tract colonization of bacteria and only include papers describing symptomatic urinary tract infections. Finally, 
we will assess the completeness using the combined checklist of STROBE criteria of the observational studies. In addition, we will evaluate the quality of the papers using the Newcastle-Ottawa Scale.

We anticipate that this paper will bring forth valuable insights in mapping bacterial maternal infection/sepsis and AMR in SSA for global health consideration. 


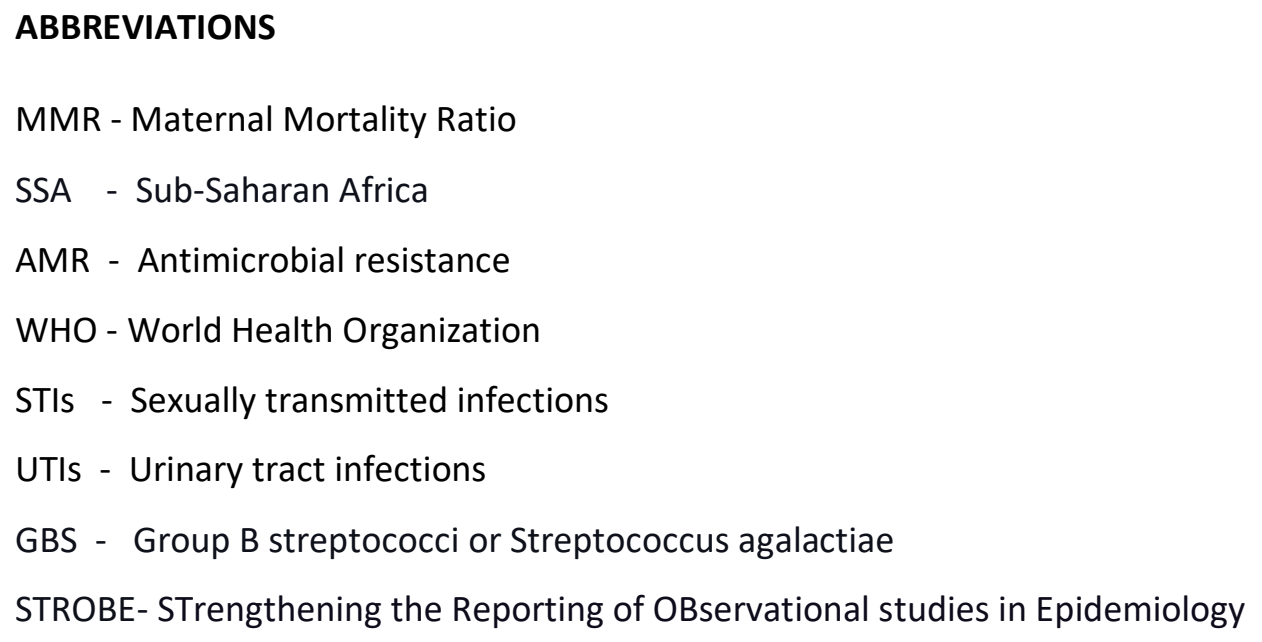




\section{DECLARATIONS}

\section{Ethics approval and consent to participate}

Not required.

\section{Consent for publication}

Not required.

\section{Availability of data and materials}

N/A

\section{Competing interests}

None to declare

\section{Funding}

The sponsor for this project is the Liverpool School of Tropical Medicine and it was funded by the Wellcome Trust. This work was supported by the MLW Programme Core Award (WTT 803) from the Wellcome Trust.

\section{Authors' contributions}

DL is the guarantor of this manuscript. DL, CC and CVD developed the research question. CC, CVD and AS devised the search strategy. DL, EM, NF, CD and DK made substantial contributions to the study design. CC and CVD drafted the manuscript. All authors have read and approved the final manuscript. 
Version 1.0 


\section{REFERENCES}

1. WHO, UNICEF, UNFPA WBG and the UNPD. WHO | Trends in maternal mortality: 1990 to 2015 [Internet]. WHO. 2016 [cited 2021 Aug 26]. p. 18-25. Available from:

https://www.who.int/reproductivehealth/publications/monitoring/maternal-mortality-2015/en/

2. Chatterjee DK. United Nations Millennium Development Goals. In: Encyclopedia of Global Justice. United Nations; 2011. p. 1105-1105.

3. UNSD, DESA. SDG Indicators - SDG Indicators [Internet]. United Nations Statistics Division, Department of Economic and Social Affairs. 2017 [cited 2021 Aug 26]. Available from: https://unstats.un.org/sdgs/metadata/?Text=\&Goal=3\&Target=3.1

4. Bonet M, Brizuela V, Abalos E, Cuesta C, Baguiya A, Chamillard M, et al. Frequency and management of maternal infection in health facilities in 52 countries (GLOSS): a 1-week inception cohort study. Lancet Glob Heal [Internet]. 2020 May 1 [cited 2021 Jan 4];8(5):e661-71. Available from: www.thelancet.com/lancetgh

5. Chou D, Daelmans B, Jolivet RR, Kinney M, Say L. Ending preventable maternal and newborn mortality and stillbirths. BMJ [Internet]. 2015 [cited 2021 Aug 26];351:19-22. Available from: www.who.int/reproductivehealth

6. UNICEF. Maternal mortality rates and statistics - UNICEF DATA [Internet]. UNICEF Data: Monitoring the situation of children and women. 2019 [cited 2021 Oct 25]. p. 1-8. Available from: https://data.unicef.org/topic/maternal-health/maternal-mortality/

7. Say L, Chou D, Gemmill A, Tunçalp Ö, Moller AB, Daniels J, et al. Global causes of maternal death: A WHO systematic analysis. Lancet Glob Heal [Internet]. 2014 Jun 1 [cited 2021 Aug 26];2(6):e323-33. Available from: http://www.thelancet.com/article/S2214109X1470227X/fulltext

8. Iskandar K, Molinier L, Hallit S, Sartelli M, Hardcastle TC, Haque M, et al. Surveillance of antimicrobial resistance in low- and middle-income countries: a scattered picture [Internet]. Vol. 10, Antimicrobial Resistance and Infection Control. BioMed Central; 2021 [cited 2021 Aug 26]. p. 1-19. Available from: https://aricjournal.biomedcentral.com/articles/10.1186/s13756-021- 
00931-w

9. Singer M, Deutschman CS, Seymour C, Shankar-Hari M, Annane D, Bauer M, et al. The third international consensus definitions for sepsis and septic shock (sepsis-3) [Internet]. Vol. 315, JAMA - Journal of the American Medical Association. American Medical Association; 2016 [cited 2021 Aug 26]. p. 801-10. Available from:

https://jamanetwork.com/journals/jama/fullarticle/2492881

10. Bonet M, Nogueira Pileggi V, Rijken MJ, Coomarasamy A, Lissauer D, Souza JP, et al. Towards a consensus definition of maternal sepsis: results of a systematic review and expert consultation [Internet]. Vol. 14, Reproductive Health. BioMed Central; 2017 [cited 2021 Aug 26]. p. 1-13. Available from: https://reproductive-healthjournal.biomedcentral.com/articles/10.1186/s12978-017-0321-6

11. Lee JH, Cho HK, Kim KH, Kim CH, Kim DS, Kim KN, et al. Etiology of invasive bacterial infections in immunocompetent children in Korea (1996-2005): A retrospective multicenter study. J Korean Med Sci [Internet]. 2011 [cited 2021 Dec 20];26(2):174-83. Available from: https://www.ncbi.nlm.nih.gov/pmc/articles/PMC3030999/

12. Okomo U, Akpalu ENK, Le Doare K, Roca A, Cousens S, Jarde A, et al. Aetiology of invasive bacterial infection and antimicrobial resistance in neonates in sub-Saharan Africa: a systematic review and meta-analysis in line with the STROBE-NI reporting guidelines. Lancet Infect Dis. 2019 Nov 1;19(11):1219-34.

13. Zlowodzki M, Poolman RW, Kerkhoffs GM, Tornetta P, Bhandari M. How to interpret a metaanalysis and judge its value as a guide for clinical practice. Acta Orthop [Internet]. 2007 Oct 1 [cited 2021 Aug 26];78(5):598-609. Available from: https://www.tandfonline.com/doi/abs/10.1080/17453670710014284

14. Raabe VN, Shane AL. Group B Streptococcus ( Streptococcus agalactiae ). Microbiol Spectr [Internet]. 2019 Apr 12 [cited 2021 Aug 26];7(2). Available from: /pmc/articles/PMC6432937/

15. Steer PJ, Russell AB, Kochhar S, Cox P, Plumb J, Rao GG. Group B streptococcal disease in the mother and newborn-A review. Eur J Obstet Gynecol Reprod Biol [Internet]. 2020 Sep 1 [cited 2021 Aug 26];252:526. Available from:/pmc/articles/PMC7295463/

Version 1.0 
Version 1.0 
Version 1.0 
Version 1.0 
Version 1.0 
Version 1.0 


\section{Supplementary Files}

This is a list of supplementary files associated with this preprint. Click to download.

- V1.0ManuscriptSRProtocolBMC201221supplementary.pdf 